\title{
Evaluation of apolipoprotein $A-I I$ as a positional candidate gene for familial Type II diabetes, altered lipid concentrations, and insulin resistance
}

\author{
S. C. Elbein ${ }^{1}$, W. Chu ${ }^{1}$, Q. Ren ${ }^{1}$, H. Wang ${ }^{1}$, C. Hemphill ${ }^{1}$, S. J. Hasstedt ${ }^{2}$ \\ ${ }^{1}$ Central Arkansas Veterans Healthcare System and Department of Medicine, University of Arkansas for Medical Sciences, \\ Little Rock, Arkansas, USA \\ ${ }^{2}$ Department of Human Genetics, University of Utah School of Medicine, Salt Lake City, Utah, USA
}

\begin{abstract}
Aims/hypothesis. We hypothesized that apolipoprotein $A$-II sequence variation was responsible for the observed linkage of Type II (non-insulin-dependent) diabetes mellitus to the apolipoprotein A-II region in Northern European families ascertained for multiple diabetic siblings, and might also influence insulin sensitivity and secretion, non-esterified fatty acids, and lipids.

Methods. We recruited 698 members of 63 families for pedigree studies and additional unrelated people providing 117 diabetic and 130 control subjects. We screened the apolipoprotein $A-I I$ gene by single strand conformation polymorphism analysis and fluorescent sequence analysis. Variants were typed by oligonucleotide ligation assay, restriction digest of amplification products, or radioactive fragment analysis for the microsatellite polymorphism. Association of each variant with Type II diabetes was tested in the case-control population by chi-square analysis, or using transmission disequilibrium test in families. Haplotypes were established in families using SIMWALK and

tested for association with diabetes and quantitative traits.

Results. No detected variant altered the coding sequence of the gene. Three single nucleotide polymorphisms showed modest evidence for an association, but no variant or haplotype was associated with diabetes in families. Similarly, we found no association with non-esterified fatty acid concentrations, HDL concentrations, or fasting insulin. In contrast, we found evidence for an association of some haplotypes and individual variants with 2 -h post-challenge glucose and measures of insulin secretion.

Conclusion/interpretation. Apolipoprotein A-II is not likely to explain the observed linkage of Type II diabetes, but variation in this gene could alter insulin secretion and post-challenge glucose. [Diabetologia (2002) 45:1026-1033]

Keywords Apolipoprotein A-II, association study, insulin sensitivity, non-esterified fatty acids, insulin secretion, transmission disequilibrium test, Type II diabetes mellitus, single nucleotide polymorphism, single stranded conformational polymorphism, haplotypes.
\end{abstract}

Received: 12 November 2001 / Revised: 6 March 2002

Published online: 8 May 2002

(C) Springer-Verlag 2002

Corresponding author: S. C. Elbein, MD, Endocrinology 111J/LR, John L. McClellan Memorial Veterans Hospital, 4300 West $7^{\text {th }}$ St., Little Rock, AR 72205, USA, e-mail: elbeinstevenc@uams.edu

Abbreviations: AIR, ${ }_{g}$ Acute insulin response to glucose; APOA2, apoliprotein Å2 gene and locus; OCI60, oral compensation index at 60 min glucose; PAP, pedigree analysis package; $S{ }_{I}$ insulin sensitivity index; SNP, single nucleotide polymorphism; SSCP, single strand conformation polymorphism analysis; TDT, transmission disequilibrium test; df, degrees of freedom.

Considerable data support a strong genetic component to Type II (non-insulin-dependent) diabetes mellitus, but the genetic predisposition is probably complex with multiple susceptibility loci interacting with each other and with environmental and life-style factors. Recently genome scans from our laboratory in Caucasian families from Utah [1] and independent scans in Pima Indians [2], French Caucasian families [3], British Caucasian sib pairs [4], and Amish families [5] (A. Shuldiner, personal communication) have mapped one locus to chromosome 1q21-q23. Utah, Pima, and French studies all show peak linkage to the apolipo- 
protein A2 locus (APOA2). Although multiple positional candidate genes exist within the confidence interval for linkage, considerable data support the APOA2 gene as a functional candidate in addition to its location within a well replicated region of linkage. APOA2 was linked to non-esterified fatty acid concentrations (NEFA) in mice and humans [6]. Increased NEFA concentrations have been shown to decrease insulin action in muscle [7], increase hepatic glucose output [8], and possibly alter insulin secretion [9, 10, 11]. A common polymorphism in the $3^{\prime}$ flanking region of the APOA2 gene has been associated with increased APOA2 serum levels, coronary risk, and altered lipid profiles [12, 13, 14, 15, 16, 17].

Mouse models provide additional evidence that the APOA2 locus might alter insulin sensitivity. Heterozygous and homozygous mice made deficient in apolipoprotein A-II by homologous recombination showed profound decreases in HDL cholesterol, reduced NEFA concentrations, reduced glucose, and low insulin concentrations, thus suggesting improved insulin sensitivity [18]. In contrast, transgenic mice overexpressing APOA2 have increased atherogenesis [19, 20], evidence for insulin resistance with higher fasting insulin concentrations and delayed clearance of a glucose bolus, increased obesity, increased triglycerides, and increased NEFA concentrations [21]. Thus, mice overexpressing and underexpressing APOA2 show altered insulin sensitivity and glucose homeostasis, consistent with a direct or indirect role for APOA2 in diabetes risk.

Because the APOA2 locus can be implicated in Type II diabetes susceptibility by linkage studies with Type II diabetes mellitus, linkage to NEFA concentrations, and a role in glucose homeostasis in experimental animal models, we evaluated the role of common variants in this gene in members of Caucasian families ascertained for at least two diabetic siblings. We screened the exons and flanking regions for novel variants in family members and control subjects, and evaluated the common variants for association with Type II diabetes in both case-control and family-based studies. We also evaluated the role of these common variants in glucose homeostasis, control of NEFA concentrations, and HDL and triglyceride metabolism.

\section{Subjects and methods}

Study population. All subjects studied were of Northern European ancestry and were ascertained in Utah under a protocol approved by the University of Utah Institutional Review Board. All subjects provided informed consent. Family based studies included members of 63 families whose characteristics have been described $[1,22,23]$. Genotypic data were available on up to 698 people, including 283 affected, 388 unaffected, and 27 subjects who were considered to have an unknown diagnosis. Non-diabetic family members were characterized by glucose and insulin concentrations during a standard $75 \mathrm{gm}$
OGTT. Non-esterified fatty acids concentrations were available on a subset of 475 family members, mostly from families ascertained later in the study. Direct measures of insulin sensitivity and secretion derived from the tolbutamide-modified frequently sampled intravenous glucose tolerance tests were available for 126 members of 26 kindreds [23, 24]. Case-control studies were conducted on 117 unrelated, non-diabetic control subjects and 130 unrelated diabetic patients, of whom 69 were unrelated members of families that met the criteria of two diabetic siblings. Not all diabetic family members were included in earlier linkage studies [1].

Mutation detection and typing. We selected 25 unrelated diabetic family members from families with the greatest evidence for linkage to chromosome 1q21-q23 and 10 unrelated control subjects from the same population. Primers were designed to amplify six fragments covering the region from $430 \mathrm{bp}$ upstream of exon 1 to $527 \mathrm{bp}$ of the $3^{\prime}$ flanking region, along with intron sequences flanking the exons. Mutations were detected by single strand conformation polymorphism analysis (SSCP) under five nondenaturing conditions on fragments of under 350 bp: $5 \%$ polyacrylamide with and without $10 \%$ glycerol and at room temperature or at $4{ }^{\circ} \mathrm{C}$, and mutation detection enhancement gels (MDE; FMC Bioproducts, Rockland, Me., USA) at room temperature. We used bidirectional fluorescent sequencing [25] on a LI-COR GR4200 (LI-COR, Lincoln, Neb., USA) to confirm all variants and to check the sensitivity of our SSCP screening in 16 diabetic patients. Finally, single nucleotide polymorphisms (SNPs) were searched for using the published databases [26, 27] and literature, and confirmed using SSCP and sequence analysis.

The microsatellite polymorphism in intron 2 was typed radioactively [1]. SNPs at locations -262, 904, and 1099 were typed using an oligonucleotide ligation assay, with radioactive detection on a Storm 860 Phosphoimager (Molecular Dynamics, Sunnyvale, Calif., USA) after polyacrylamide gel separation. Primer sequences for SNPs 1, 2 and 3 are shown (Table 1). For semi-automated scoring we used GeneProfiler software (Scanalytics, Fairfax, Va., USA) on digitized images. The SNP at position 1860 (SNP 4) was typed using restriction enzyme Msp I and the fragments detected on agarose gels [17].

Statistical analysis. Association of each SNP with Type II diabetes was tested in the unrelated population by chi-square analysis of allelic association or by Fisher's Exact test [28]. Additionally, based on the prior evidence for a recessive locus [1], we tested homozygotes for each allele against homozygotes and heterozygotes for the other allele. Family based associations with Type II diabetes were tested using a modified transmission disequilibrium test (TDT) [29] which used likelihood analysis and full pedigree data to probabilistically infer missing parental genotypes and haplotypes. The analysis compared the model with a maximised likelihood to the likelihood of no increased transmission $(\tau=0.5)$. The likelihoods of the genetic models [30] were computed using the Pedigree Analysis Package [31] and the maxima were obtained using GEMINI [32]. Hypothesis testing compared the likelihood of a submodel ( $\tau=0.50$; null hypothesis) to the likelihood of a more general model (transmission maximised). Twice the natural logarithm of the ratio of the likelihoods has an asymptotic chi-square distribution. The allele frequency of each SNP was estimated from unrelated pedigree members, assuming Hardy-Weinberg equilibrium, but using genotypes on all pedigree members to identify when individuals had not been genotyped.

Haplotypes were derived from all family data using the program SIMWALK2 version 2.60 [33]. For haplotype analysis, we included the nine observed alleles of the microsatellite polymorphism. Haplotype frequencies were estimated similar- 
Table 1. Genotyping primers for APOA2 single nucleotide polymorphisms by oligonucleotide ligation assay

\begin{tabular}{|c|c|c|}
\hline SNP & Allele specific primers & Common primer \\
\hline SNP1 $(-262)$ & $\begin{array}{l}\text { CTTGGAATCCTGCTTCCTGTTGCGT } \\
\text { TATTCTTGGAATCCTGCTTCCTGTTGCGC }\end{array}$ & TGAGAGGCAGGGGGTACAGCTCTTA \\
\hline SNP2 (904) & $\begin{array}{l}\text { AACAGCAAGAACTGGGCCTGG } \\
\text { TCTTAACAGCAAGAACTGGGCCTGC }\end{array}$ & GTGGGAGGTCAGAGCAGACTG \\
\hline SNP3 (1099) & $\begin{array}{l}\text { AAACTCTAATCCCCTCACCCTATC } \\
\text { CTAGAAACTCTAATCCCCTCACCTATT }\end{array}$ & GTTCCGTTCCAGCCTTCTTGATC \\
\hline
\end{tabular}

Primer sequences for oligonucleotide ligation assays. Conditions are available from the authors. Numbers in parentheses refer to the location relative to the start of exon 1 (see Table 2 )

ly by using PAP and assuming tight linkage between the SNPs, and haplotype transmission was tested using a transmission disequilibrium test as described above. We combined all rare haplotypes into a single haplotype 9. We used likelihood analysis to compute the probability of the correct genotype assignment for each subject.

For quantitative trait analyses, we tested only non-diabetic subjects for insulin, glucose, and NEFA, and we tested both non-diabetic subjects and all subjects for triglycerides and HDL cholesterol. All skewed measures, including insulin, glucose, BMI, and lipid measures were log-transformed to normality and adjusted for age and sex prior to analysis. An effect of each SNP on the quantitative traits was tested using a measured genotype analysis in PAP. A mean for each genotype was estimated and tested against a single mean in a two degrees of freedom (df) chi-square test. An effect of the microsatellite or haplotype on the quantitative traits was tested using measured allele analysis in PAP. Effects of each allele were estimated and tested against no effect of any allele ( $9 \mathrm{df}$ ) or no effect of a single allele $(1 \mathrm{df})$. Polygenic inheritance was included for both measured genotype and measured allele analysis. Analysis of subjects who had undergone frequently sampled intravenous glucose tolerance tests was done using mixed effects linear regression analysis in SPSS v 10.1 (SPSS, Chicago, Ill., USA) [34]. Family membership was a random factor, genotype and diagnosis (normal and IGT) were fixed factors, and age and ln-transformed BMI were covariates, as we have described previously [35]. BMI, insulin sensitivity index $\left(\mathrm{S}_{\mathrm{I}}\right)$, acute insulin response to glucose $\left(\mathrm{AIR}_{\mathrm{g}}\right)$, and disposition index $\left(\mathrm{S}_{\mathrm{I}}^{*} \mathrm{AIR} \mathrm{g}_{\mathrm{g}}\right)$ [36] were all ln-transformed prior to analysis. This analysis provides results comparable to that conducted in PAP to account for exact pedigree relationships [35]. Because many of the tests were correlated, and because of prior evidence for a gene in this region, we present $p$ values without correction for multiple testing.

To examine a parameter comparable to disposition index in a larger group of subjects, we derived an index which we call the "oral compensation index" (OCI). We used the 60 min insulin and glucose to measure insulin secretion, as these are most widely available in our population, and derived the $60 \mathrm{~min}$ oral compensation index (OCI60) as $\mathrm{OCI}_{60}=$ $\left.\ln \left[\left(\mathrm{I}_{60}-\mathrm{I}_{0}\right) / \mathrm{G}_{60}\right]+\ln \left[1 / \mathrm{I}_{0} * \mathrm{G}_{0}\right]\right)$, where $\mathrm{I}_{0}$ and $\mathrm{I}_{60}$ are baseline and 60 min insulin concentrations, and $\mathrm{G}_{0}$ and $\mathrm{G}_{60}$ are baseline and 60 min glucose concentrations. The correlation with disposition index is 0.42 .

Linkage disequilibrium was calculated after establishing haplotypes using gene-counting and the expectation maximisation (E-M) algorithm [37] using the EH and 2LD computer programs [38].

\section{Results}

The APOA2 gene comprises four exons and three introns (Fig. 1). In contrast to the published reports, which predict at least 10 SNPs, we identified only three SNPs by a combination of detection methods: a $\mathrm{T} / \mathrm{C}$ at position -262 in the $5^{\prime}$ flanking region (SNP1); a G/C substitution at position 904 in intron 3 (SNP2); and a C/T substitution at position 1099 in intron 3 (SNP3) (all locations are given relative to exon 1 start

Fig. 1. Map of Apolipoprotein A2 Gene. The figure shows the approximate location of the four single nucleotide polymorphisms identified for this analysis, the GT repeat microsatellite, and the four exons. All numbers are relative to the transcription start site; $5^{\prime}$ flanking polymorphisms are denoted as negative numbers. Distances were taken from GenBank accession XO4898. The four SNPs are labelled as in Tables 1 and 2
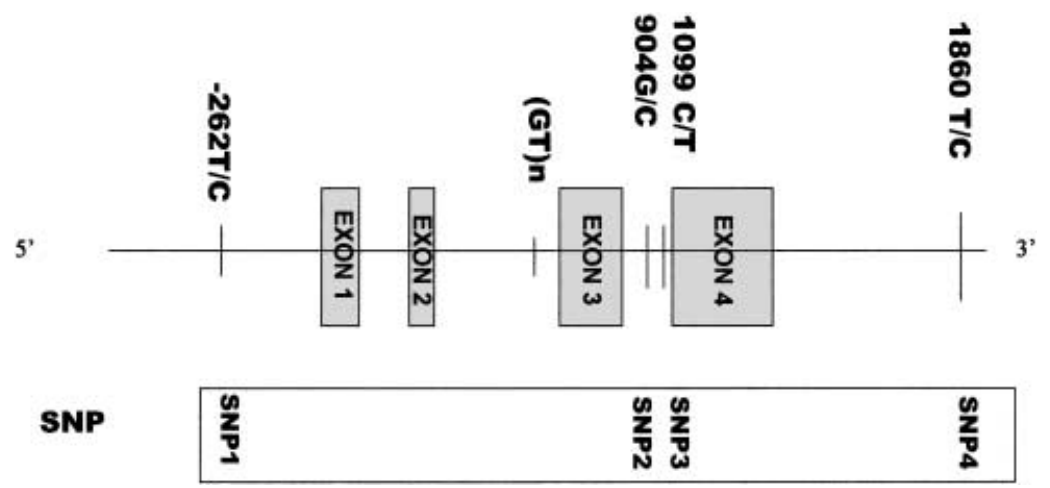
Table 2. Allelic association of APOA2 single nucleotide polymorphisms and diabetes in unrelated subjects

\begin{tabular}{llllllll}
\hline Polymorph & Location & Freq, FT2DM & Freq, T2DM & Freq, Control & $p$, FT2DM & $p$, T2DM & Relative risk \\
\hline SNP1 & -262 T/C & 0.500 & 0.602 & 0.593 & 0.051 & 0.434 & $0.69(0.45,1.05)$ \\
SNP2 & 904 G/C & 0.883 & 0.883 & 0.794 & 0.139 & 0.239 & $1.43(0.81,2.53)$ \\
SNP3 & 1099 C/T & 0.938 & 0.924 & 0.872 & 0.036 & 0.157 & $2.23(0.97,5.14)$ \\
SNP4 & 1860 C/T & 0.098 & 0.150 & 0.189 & 0.030 & 0.291 & $0.47(0.23,0.97)$ \\
\hline
\end{tabular}

Freq, FT2DM: allele frequency in members of familial kindreds with Type II (non-insulin-dependent) diabetes mellitus (T2DM); Freq, T2DM, allele frequency in all diabetic subjects; Freq, Control: frequency in unrelated non-diabetic subjects; $p$, FT2DM: significance on $2 \mathrm{X} 2 \chi^{2}$ analysis, diabetic family mem-

Table 3. Linkage disequilibrium between APOA2 single nucleotide polymorphisms

\begin{tabular}{llrll}
\hline & SNP1 & \multicolumn{1}{c}{ SNP2 } & SNP3 & SNP4 \\
\hline SNP1 & - & $-1.000^{\mathrm{a}}$ & $-0.758^{\mathrm{b}}$ & $1.000^{\mathrm{a}}$ \\
SNP2 & - & $-0.711^{\mathrm{d}}$ & $-1.000^{\mathrm{c}}$ & \\
SNP3 & - & $1.000^{\mathrm{a}}$ & & \\
SNP4 & - & & & \\
\hline
\end{tabular}

All pairwise disequilibrium statistics are significant, as noted: ${ }^{\mathrm{a}} p<0.000001,{ }^{\mathrm{b}} p<0.0001,{ }^{\mathrm{c}} p<0.01,{ }^{\mathrm{d}} p<0.05$

Linkage disequilibrium statistic $\left(D^{\prime}\right)$ is shown for each biallelic SNP from 5' (SNP1) to 3' (SNP4), as shown in Figure 1

site). The T/C substitution that altered an Msp1 site at position 1860 in the $3^{\prime}$ flanking region was beyond the region we selected for screening but has been described well before. All SNPs detected have been reported previously [27, 39], and none alter the coding sequence. In addition, we identified the known polymorphic microsatellite $\left(\mathrm{GT}_{\mathrm{n}}\right)$ repeat seven nucleotides upstream of exon 3 in intron 2. The location of each SNP and the GT repeat are shown (Fig. 1).

Although we found nominal associations $(p \leq 0.05)$ for SNPs 1, 3 and 4 when unrelated diabetic family members were compared with control subjects, this significance was lost when all available unrelated diabetic patients were examined (Table 2). The estimated relative risks were approximately two for the risk allele for each variant (Table 2) but the confidence intervals were wide and not significant, consistent with the marginal $p$ values. The four SNPs were in substantial but incomplete linkage disequilibrium (Table 3), consistent with the short distance between the most $5^{\prime}$ and most 3' SNP. The lack of significant linkage disequilibrium between SNPs 2 and 3 might represent a recombination hotspot introduced by the microsatellite repeat [40].

Based on the strength of the APOA2 gene as a positional candidate and the suggestion of an association for SNPs 1, 3 and 4 among family members, we typed the four SNPs and the microsatellite polymorphism in all family members from our initial linkage study, regardless of original evidence for linkage to this re- bers vs control subjects; $p$, T2DM: significance for all diabetic patients vs control subjects. Relative risk is shown for allele 1 for diabetic members of familial T2DM kindreds vs control subjects, with $95 \% \mathrm{CI}$ in parentheses. Location is relative to the start of exon 1

gion. We tested each variant independently and as haplotypes for excess transmission to diabetic family members using the transmission disequilibrium test. We observed 34 of the 160 possible haplotypes, of which only 8 haplotypes were present in 10 or more diabetic patients. Haplotypes 1 and 2 accounted for $55 \%$ of all subjects (Table 4). This relative lack of haplotype diversity is consistent with the strong linkage disequilibrium observed in pairwise analysis (Table 3). No single SNP was over-transmitted to diabetic patients. We noted trends to over-transmission of microsatellite alleles $1(p=0.021)$ and $4(p=0.051)$. Only haplotype 9 (the combined rare haplotypes) showed evidence of over-transmission at $p=0.039$. Given that we tested nine haplotypes, nine microsatellite alleles, and three SNPs, these values clearly would not be significant after Bonferroni correction.

Given the prior evidence for a role of APOA2 in insulin sensitivity, HDL concentrations, and NEFA concentrations [6, 21, 41, 42, 43], we examined the effect of APOA2 variation and haplotypes on ten related traits: NEFA, HDL cholesterol, triglycerides, fasting insulin, fasting glucose, 60 and 120 min glucose during an OGTT and a measure of insulin secretion at $60 \mathrm{~min}$ that is normalized for the insulin sensitivity (OCI60; see Methods). Surprisingly, we found little evidence for an effect of either individual variants or haplotypes on triglycerides, HDL cholesterol, NEFA, or fasting insulin. Microsatellite allele 4 and haplotype 2 showed marginal effects on triglyceride concentrations ( $p=0.029$ and $p=0.046$, respectively) and alleles 7 and 8 showed a marginal effect on NEFA ( $p=0.035$ and $p=0.046$, respectively), but none would be significant after Bonferroni correction. We found a trend to effects on fasting glucose for microsatellite allele 1 and haplotype 5 (Table 4$)(p=0.049$ and $p=0.003$, respectively), and evidence for an effect of APOA2 on 120 min glucose during the OGTT, with SNP1 (promoter region), microsatellite allele 1, SNP 2, SNP4, and haplotypes 1 and 3 all showing evidence for an association (Table 5 ).

Among 113 subjects from 26 families who were both successfully genotyped and who had undergone intravenous glucose tolerance tests with Minimal 
Table 4. Haplotypes observed in at least ten diabetic patients

\begin{tabular}{lllllllll}
\hline Assignment & 1 & 2 & 3 & 4 & 5 & 6 & 7 & 8 \\
\hline Haplotype & 26112 & 13112 & 11211 & 12122 & 16122 & 12211 & 27112 & 15112 \\
Frequency & 0.325 & 0.225 & 0.093 & 0.076 & 0.035 & 0.030 & 0.032 & 0.024 \\
\hline
\end{tabular}

The table shows the eight haplotypes that were observed in ten or more diabetic family members with the population frequency estimated from the family data. The major allele for each single nucleotide polymorphism is denoted as 1 and the minor allele

Table 5. Significant measured genotype and allele effects for SNPs, microsatellite alleles, and haplotypes for quantitative traits

\begin{tabular}{llll}
\hline Variant & Trait & Number & $p$ value \\
\hline Microsatellite allele 1 & fasting glucose & 429 & 0.049 \\
Haplotype 5 & fasting glucose & 331 & 0.003 \\
SNP3 (1099) & 60 min glucose & 360 & 0.012 \\
SNP1 (-292) & 120 min glucose & 331 & 0.008 \\
SNP2 (904) & 120 min glucose & 349 & 0.003 \\
SNP4 (1860) & 120 min glucose & 335 & 0.029 \\
Microsatellite allele 1 & 120 min glucose & 418 & 0.015 \\
Haplotype 1 & 120 min glucose & 323 & 0.017 \\
Haplotype 3 & 120 min glucose & 323 & 0.008 \\
Microsatellite allele 1 & OCI60 & 395 & 0.003 \\
Microsatellite allele 5 & OCI60 & 395 & 0.010 \\
Haplotype 3 & OCI60 & 302 & 0.039 \\
Haplotype 4 & OCI60 & 302 & 0.038 \\
\hline
\end{tabular}

Traits showing significant $(p<0.05)$ association with single nucleotide polymorphisms (SNPs), individual microsatellite alleles, or haplotypes. Number, number of non-diabetic subjects tested; OCI60, oral compensation index at $60 \mathrm{~min}$, as defined in the Methods. $p$ values are shown without Bonferroni correction for multiple tests, since the tests are not fully independent

Model analysis [23, 24], no variant altered insulin sensitivity $\left(\mathrm{S}_{\mathrm{I}}\right)$. We found marginal effects $(p=0.030)$ of SNP3 on first phase insulin response (acute insulin response to glucose, or $\mathrm{AIR}_{\mathrm{G}}$ ), and some evidence for an effect of SNP2 and SNP4 on disposition index $\left(\mathrm{S}_{\mathrm{I}}^{*} \mathrm{AIR}_{\mathrm{G}}\right)$, at $p=0.032$ and $p=0.034$, respectively, with interactions with family membership showing more significance $(p<0.001)$. To further explore these suggestive effects of APOA2 on insulin secretion in response to insulin sensitivity in a larger sample, we used a derived index of insulin secretion that is analogous to the disposition index from the OGTT data (OCI60, see Methods). In support of the smaller intravenous glucose tolerance test study, two microsatellite alleles and two haplotypes showed evidence for an association with OCI60 (Table 5 ).

\section{Discussion}

Linkage data in mice and humans [6], in experimental mouse models [43], transgenic mouse models [21, 41] and knockout [43] mouse models all support a role for as 2 . The microsatellite alleles were numbered $1-10$, but allele 9 was not observed in this study. The order of the individual markers in the haplotype was SNP1, APOA2 microsatellite, SNP2, SNP3, and SNP4 (see Fig. 1)

APOA2 in HDL and NEFA metabolism and in insulin sensitivity. Such a pleiotropic effect is consistent with the hypothesis that increased NEFA alter insulin sensitivity, increase hepatic glucose production, and can alter insulin secretion $[44,45]$. Human studies also support an association of $A P O A 2$ variation with atherosclerosis or hypertriglyceridaemia $[12,17,46]$. Finally, linkage of both Type II diabetes [1, 2, 3, 4] and familial combined hyperlipidaemia $[47,48]$ to the region of chromosome 1q21-q23 that includes the APOA2 locus would potentially support APOA2 as a candidate for both disorders. Studies from our laboratory found linkage in 42 multigenerational Caucasian families under a recessive model that reached genome-wide significance $(p<0.05$ for the genome wide scan, and logarithm of odds score of 4.29) with a peak at this locus [1].

Based on these data, we carefully screened the APOA2 gene for new variants in a population showing linkage of this region to Type II diabetes and examined the association with diabetes and with a number of diabetes related traits. No variation was identified within the coding sequence and no variant would be expected to alter the protein. We tested the four SNPs identified in addition to the microsatellite (simple tandem repeat) variant that sits within intron 2, very close to the upstream splice acceptor site of exon 3. Although we find marginal evidence for an association when we compare diabetic members of families used for the linkage study with control subjects from the same population, this significance is lost when we include a larger sample of diabetic patients from this same population. This finding might be expected if the families selected for multiple diabetic siblings were different from other diabetic patients in the population and if the true variant were in a region that we did not test. However, using haplotypes constructed from other family members and the transmission disequilibrium test (TDT) implemented in families [29], we found little evidence that either individual variants or haplotypes were transmitted from parents to their diabetic offspring more often than expected by chance alone (equal transmission of both alleles from heterozygous parents). We have over $70 \%$ power (depending on the allele frequency) to detect a $15 \%$ increase in transmission of an allele to affected family members under an additive model. This increased transmission 
is equivalent to a relative risk of diabetes of 1.3 for those carrying one copy of the susceptibility allele. Nonetheless, this test could have limitations in a recessive disease, particularly if a combination of two haplotypes contributes to the risk, as has been proposed for the NIDDM 1 or calpain 10 gene on chromosome $2 \mathrm{q}$ [49]. Nonetheless, APOA2 variation does not seem likely to account for the linkage to this region.

Based on the previous human and animal studies, we also tested for an association of both individual variants and haplotypes with diabetes and lipid associated traits. We were only able to show modest evidence for an association of microsatellite alleles 7 and 8 with NEFA concentrations and microsatellite allele 4 and haplotype 2 with triglyceride concentrations and we found no evidence for an association of any variant with HDL cholesterol. These findings seem to contrast the earlier linkage studies of NEFA [6]. The association findings are consistent with our inability to show linkage of these traits to this region using variance component quantitative trait linkage analysis. Our inability to replicate the earlier linkage is not surprising. Replication of linkage studies has been difficult to do and might require a much larger study than ours [28]. Furthermore, these families were ascertained for two or more diabetic siblings and thus represent a different population than the earlier study. However, none of the common variants seem likely to affect triglyceride, NEFA or HDL cholesterol concentrations in this population. Furthermore, we were not able to show an effect on insulin sensitivity, either using fasting insulin among non-diabetic subjects, homeostatic model-derived insulin sensitivity [50], or the minimal model-derived insulin sensitivity index in a subset of subjects who underwent intravenous glucose tolerance testing.

Several findings in this study were surprising and suggest a role for this gene in the contribution to diabetes susceptibility apart from our failure to reject the null hypothesis convincingly regarding the association with diabetes. Firstly, the common microsatellite allele 1 and the haplotype 5 were associated with fasting glucose, albeit with marginal significance $(p=0.049)$ in the first case. Secondly, this same microsatellite allele, SNP2, and SNP4 (the previously described Exon 4 variant) were all associated with the glucose concentration $2 \mathrm{~h}$ after a $75 \mathrm{~g}$ oral glucose load, with significance of $p=0.003$ to $p=0.029$, and haplotypes 1 and 3 were similarly associated $(p=0.017$ and $p=0.008$, respectively). We have analysed ten traits for five variants (four SNPs and the microsatellite), but neither the traits (insulin and HOMA, 60 min glucose and OCI60) nor the variants can be considered independent. Thus, a Bonferroni correction for 50 tests, which would require a $p$ value of 0.001 for a nominal significance of 0.05 , is overly conservative and might result in the rejection of a real effect. This is particularly true for $2-\mathrm{h}$ glucose and 60-min glucose, where several tests show a trend and microsatellite allele 1 and SNPs 1 and 2 are very close to the corrected $p$ value.

An additional finding was the association of SNP2 and SNP4 with disposition index. We have shown previously that disposition index is heritable in these families [23] and $A P O A 2$ variation could contribute to this polygenic heritability. Further support for this finding comes from our exploration of the OCI60 index, which is analogous to the ln-transformed disposition index but derived from the OGTT (see Methods). Using this index, we again find modest associations of all microsatellite alleles, the specific microsatellite alleles 1 and 5, and haplotypes 3, 4, and 8. Taken together, the findings of an association of APOA2 variation with insulin secretion and post-challenge glucose might suggest that the beta-cell response to insulin resistance is impaired, leading to increased 2-h glucose after a glucose load. However, the overlap between these traits and the associated variants is incomplete, and no single variant accounts for these findings.

The variant most likely to explain our findings could be the microsatellite (tandem repeat). This dinucleotide repeat ends only seven nucleotides upstream from the intron 2 splice acceptor site and the beginning of exon 3 . We have observed previously ten alleles, of which only nine were observed among the family members included in this study. Microsatellite allele 1 is associated with 120 min glucose, OCI60, and is present on haplotype 3 that also shows an association with these traits. However, no consistent association of a size range of alleles is apparent in our study, and the limited haplotype diversity suggests that the microsatellite allele might be in disequilibrium with another variant at some distance. A large population study that can focus on the microsatellite population and these traits could be required to determine whether susceptibility and protective alleles exist in this region.

In conclusion, we have evaluated one of the strongest candidate genes that might explain linkage to both Type II diabetes and to familial combined hyperlipidaemia. Our data suggest possible effects of APOA2 on post-challenge glucose and on insulin secretion related traits but we find little convincing evidence that $A P O A 2$ variation results in altered NEFA concentrations, altered insulin sensitivity, or measurable alterations in the risk for Type II diabetes. If this gene contributes to the observed linkage to Type II diabetes, it must do so together with other factors in this region.

Acknowledgements. We thank J. Wolford (NIDDK, Phoenix) for many helpful discussions and for sharing information about single nucleotide polymorphisms, primers, and sequence information; M. Hoffman, R. Mayorga, K. Barrett, D. Fuller, P. Yount, and K. Teng for technical support, and K. Wegner, T. Maxwell, and H. Tuckett for family ascertainment and DNA preparation. This work was supported by the Research Service 
of the Department of Veterans Affairs, a grant from the National Institutes of Health/NIDDK DK39311, a family acquisition grant from the American Diabetes Association, and by grants from National Institutes of Health/NCRR to the General Clinical Research Centers of the University of Utah (M01RR03655) and the University of Arkansas for Medical Sciences (M01RR14288).

\section{References}

1. Elbein SC, Hoffman MD, Teng K, Leppert MF, Hasstedt SJ (1999) A genome-wide search for type 2 diabetes susceptibility genes in Utah Caucasians. Diabetes 48:11751182

2. Hanson RL, Ehm MG, Pettitt DJ et al. (1998) An autosomal genomic scan for loci linked to type II diabetes mellitus and body-mass index in Pima Indians. Am J Hum Genet 63:1130-1138

3. Vionnet N, Hani EH, Dupont S et al. (2000) Genomewide search for type 2 diabetes-susceptibility genes in French whites: evidence for a novel susceptibility locus for earlyonset diabetes on chromosome 3q27-qter and independent replication of a type 2-diabetes locus on chromosome 1q21-q24. Am J Hum Genet 67:1470-1480

4. Wiltshire S, Hattersley AT, Hitman GA et al. (2001) A genomewide scan for loci predisposing to type 2 diabetes in a U.K. population (The Diabetes UK Warren 2 Repository): Analysis of 573 pedigrees provides independent replication of a susceptibility locus on chromosome 1q. Am J Hum Genet 69:553-569

5. St Jean P, Hsueh W-C, Mitchell BD et al (2000) Association between diabetes, obesity, glucose, and insulin levels in the Old Order Amish and SNPs within a region of linkage on chromosome 1p13-q23. Am J Hum Genet 67 [Supp 2]:332 (Abstract)

6. Warden CH, Daluiski A, Bu X et al. (1993) Evidence for linkage of the apolipoprotein A-II locus to plasma apolipoprotein A-II and free fatty acid levels in mice and humans. Proc Natl Acad Sci USA 90:10886-10890

7. Boden G (1997) Role of fatty acids in the pathogenesis of insulin resistance and NIDDM. Diabetes 46:3-10

8. Rebrin K, Steil GM, Getty L, Bergman RN (1995) Free fatty acid as a link in the regulation of hepatic glucose output by peripheral insulin. Diabetes 44:1038-1045

9. Boden G, Chen X, Iqbal N (1998) Acute lowering of plasma fatty acids lowers basal insulin secretion in diabetic and nondiabetic subjects. Diabetes 47:1609-1612

10. Carpentier A, Mittelman SD, Lamarche B, Bergman RN, Giacca A, Lewis GF (1999) Acute enhancement of insulin secretion by FFA in humans is lost with prolonged FFA elevation. Am J Physiol 276:1055-1066

11. Dobbins RL, Chester MW, Daniels MB, McGarry JD, Stein DT (1998) Circulating fatty acids are essential for efficient glucose-stimulated insulin secretion after prolonged fasting in humans. Diabetes 47:1613-1618

12. Civeira F, Genest J, Pocovi M et al. (1992) The MspI restriction fragment length polymorphism $3^{\prime}$ to the apolipoprotein A-II gene: relationships with lipids, apolipoproteins, and premature coronary artery disease. Atherosclerosis 92:165-176

13. Bu X, Warden CH, Xia YR et al. (1994) Linkage analysis of the genetic determinants of high density lipoprotein concentrations and composition: evidence for involvement of the apolipoprotein A-II and cholesteryl ester transfer protein loci. Hum Genet 93:639-648
14. Kessling AM, Rajput-Wiliams J, Bainton D et al. (1988) DNA polymorphisms of the apolipoprotein AII and AICIII-AIV genes: a study in men selected for differences in high-density-lipoprotein cholesterol concentration. Am J Hum Genet 42:458-467

15. Scott J, Knott TJ, Priestley LM et al. (1985) High-density lipoprotein composition is altered by a common DNA polymorphism adjacent to apoprotein AII gene in man. Lancet 1:771-773

16. Thorn JA, Stocks J, Reichl D, Alcolado JC, Chamberlain JC, Galton D (1993) Variability of plasma apolipoprotein (apo) A-II levels associated with an apo A-II gene polymorphism in monozygotic twin pairs. Biochim Biophys Acta1180:299-303

17. Vohl MC, Lamarche B, Bergeron J et al. (1997) The MspI polymorphism of the apolipoprotein A-II gene as a modulator of the dyslipidemic state found in visceral obesity. Atherosclerosis 128:183-190

18. Weng W, Brandenburg NA, Zhong S et al. (1999) ApoA-II maintains HDL levels in part by inhibition of hepatic lipase. Studies In apoA-II and hepatic lipase double knockout mice. J Lipid Res 40:1064-1070

19. Warden CH, Hedrick CC, Qiao JH, Castellani LW, Lusis AJ (1993) Atherosclerosis in transgenic mice overexpressing apolipoprotein A-II. Science 261:469-472

20. Schultz JR, Gong EL, McCall MR, Nichols AV, Clift SM, Rubin EM (1992) Expression of human apolipoprotein A-II and its effect on high density lipoproteins in transgenic mice. J Biol Chem 267:21630-21636

21. Castellani LW, Goto AM, Lusis AJ (2001) Studies with apolipoprotein A-II transgenic mice indicate a role for HDLs in adiposity and insulin resistance. Diabetes 50:643-651

22. Elbein SC, Bragg KL, Hoffman MD, Mayorga RA, Leppert MF (1996) Linkage studies of NIDDM with 23 chromosome 11 markers in a sample of whites of northern European descent. Diabetes 45:370-375

23. Elbein SC, Hasstedt SJ, Wegner K, Kahn SE (1999) Heritability of pancreatic beta-cell function among nondiabetic members of Caucasian familial type 2 diabetic kindreds. J Clin Endocrinol Metab 84:1398-1403

24. Elbein SC, Wegner K, Kahn SE (2000) Reduced beta-cell compensation to the insulin resistance associated with obesity in members of caucasian familial type 2 diabetic kindreds. Diabetes Care 23:221-227

25. Shevchenko YO, Bale SJ, Compton JG (2000) Mutation screening using automated bidirectional dideoxy fingerprinting. BioTechniques 28:134-138

26. Sherry ST, Ward MH, Kholodov M et al. (2001) dbSNP: the NCBI database of genetic variation. Nucleic Acids Res 29:308-311

27. Halushka MK, Fan JB, Bentley K et al. (1999) Patterns of single-nucleotide polymorphisms in candidate genes for blood-pressure homeostasis. Nat Genet 22:239-247

28. Ott J (1999) Analysis of Human Genetic Linkage, (3rd edn.), Johns Hopkins University Press, Baltimore and London, pp 196-198

29. Ewens WJ, Spielman RS (1995) The transmission/disequilibrium test: history, subdivision, and admixture. Am J Hum Genet 57:455-464

30. Elston RC, Stewart J (1971) A general model for the genetic analysis of pedigree data. Hum Hered 21:523-542

31. Hasstedt SJ (2001) PAP: Pedigree Analysis Package, Version 5. University of Utah, Department of Genetics, Salt Lake City, UT

32. Lalouel JM (1979) GEMINI-A computer program for optimization of general nonlinear functions. Technical Report 14, University of Utah, Department of Medical Biophysics and Computing, Salt Lake City 
33. Sobel E, Lange K (1996) Descent graphs in pedigree analysis: applications to haplotyping, location scores, and marker-sharing statistics. Am J Hum Genet 58:1323-1337

34. Allison DB, Heo M, Kaplan N, Martin ER (1999) Siblingbased tests of linkage and association for quantitative traits. Am J Hum Genet 64:1754-1763

35. Elbein SC, Sun J, Scroggin E, Teng K, Hasstedt SJ (2001) Role of common sequence variants in insulin secretion in familial type 2 diabetic kindreds: the sulfonylurea receptor, glucokinase, and hepatocyte nuclear factor 1alpha genes. Diabetes Care 24:472-478

36. Kahn SE, Prigeon RL, McCulloch DK et al. (1993) Quantification of the relationship between insulin sensitivity and beta-cell function in human subjects: evidence for a hyperbolic function. Diabetes 42:1663-1672

37. Long JC, Williams RC, Unbanek M (1995) An E-M algorithm and testing strategy for multiple-locus haplotypes. Am J Hum Genet 56:799-810

38. Xie X, Ott J (1993) Testing linkage disequilibrium between a disease gene and marker loci. Am J Hum Genet 53:1107 (Abstract)

39. Tsao YK, Wei CF, Robberson DL, Gotto AM Jr. Chan L (1985) Isolation and characterization of the human apolipoprotein A-II gene. Electron microscopic analysis of RNA: DNA hybrids, nucleotide sequence, identification of a polymorphic MspI site, and general structural organization of apolipoprotein genes. J Biol Chem 260:15222-15231

40. Chakravarti A, Elbein SC, Permutt MA (1986) Evidence for increased recombination near the human insulin gene: implication for disease association studies. Proc Natl Acad Sci USA 83:1045-1049

41. Julve J, Escola-Gil JC, Marzal-Casacuberta A, OrdonezLlanos J, Gonzalez-Sastre F, Blanco-Vaca F (2000) Increased production of very-low-density lipoproteins in transgenic mice overexpressing human apolipoprotein A-II and fed with a high-fat diet. Biochim Biophys Acta 1488:233-244
42. Boisfer E, Lambert G, Atger V et al. (1999) Overexpression of human apolipoprotein A-II in mice induces hypertriglyceridemia due to defective very low density lipoprotein hydrolysis. J Biol Chem 274:11564-11572

43. Weng W, Breslow JL (1996) Dramatically decreased high density lipoprotein cholesterol, increased remnant clearance, and insulin hypersensitivity in apolipoprotein A-II knockeout mice suggest a complex role for apolipoprotein A-II in atherosclerosis susceptibility. Proc Natl Acad Sci USA 93:14788-14794

44. Boden G (1998) Free fatty acids (FFA), a link between obesity and insulin resistance. Front Biosci 3:169-175

45. Unger RH, Zhou YT (2001) Lipotoxicity of beta-cells in obesity and in other causes of fatty acid spillover. Diabetes 50 [Suppl 1]:S118-S121

46. Hanis CL, Chakraborty R, Hewett-Emmett D (1985) How much of variability in apolipoprotein AII concentrations is explained by polymorphism adjacent to apo-AII gene? Lancet 1:1339-1340

47. Coon H, Myers RH, Borecki IB et al. (2000) Replication of linkage of familial combined hyperlipidemia to chromosome 1q with additional heterogeneous effect of apolipoprotein A-I/C-III/A-IV locus: the NHLBI family heart study. Arterioscler Thromb Vasc Biol 20:2275-2280

48. Pajukanta P, Nuotio I, Terwilliger JD et al. (1998) Linkage of familial combined hyperlipidaemia to chromosome 1q21-q23. Nat Genet 18:369-373

49. Horikawa Y, Oda N, Cox NJ et al. (2000) Genetic variation in the gene encoding calpain-10 is associated with type 2 diabetes mellitus. Nat Genet 26:163-175

50. Matthews DR, Hosker JP, Rudenski AS, Naylor BA, Treacher DF, Turner RC (1985) Homeostasis model assessment: insulin resistance and beta-cell function from fasting plasma glucose and insulin concentrations in man. Diabetologia 28:412-419 